\title{
Bounds on the Dimensions of Trivariate Spline Spaces
}

\author{
Peter Alfeld ${ }^{1)}$ and Larry L. Schumaker ${ }^{2)}$
}

\begin{abstract}
We derive upper and lower bounds on the dimensions of trivariate spline spaces defined on tetrahedral partitions. The results hold for general partitions, and for all degrees of smoothness $r$ and polynomial degrees $d$.
\end{abstract}

Keywords: Dimension, Trivariate Splines

AMS Classification: $41 \mathrm{~A} 15$

\section{$\S 1$. Introduction}

Suppose $\triangle$ is a finite collection of nondegenerate tetrahedra such that if any two tetrahedra in $\triangle$ intersect, then their intersection is exactly one vertex, one edge, or one face. Then we call $\triangle$ a tetrahedral partition of the set $\Omega$, where $\Omega$ is the union of all tetrahedra in $\triangle$. This definition of a tetrahedral partition allows $\Omega$ to have holes and cavities as well as pinch points where just two tetrahedra meet in a single point.

Given $0 \leq r \leq d$, let $\mathcal{P}_{d}$ be the $\left(\begin{array}{c}d+3 \\ 3\end{array}\right)$-dimensional space of trivariate polynomials of degree $d$. In this paper we study the space

$$
\mathcal{S}_{d}^{r}(\triangle)=\left\{s \in C^{r}(\Omega):\left.s\right|_{T} \in \mathcal{P}_{d}, \text { for all } T \in \triangle\right\}
$$

of trivariate splines of smoothness $r$ and degree $d$ associated with $\triangle$. Here the condition $s \in C^{r}(\Omega)$ means that $s$ belongs to $C^{r}(v)$ for every point $v \in \Omega$, i.e., if $v$ is contained in the tetrahedra $T_{1}, \ldots, T_{m}$, then all of the polynomials $\left.s\right|_{T_{1}}, \ldots,\left.s\right|_{T_{m}}$ have common derivatives up to order $r$ at $v$.

Trivariate splines are important tools in approximation theory and numerical analysis, and have attracted considerable interest in the past few years, see [3] and references therein.

Clearly $\mathcal{S}_{d}^{r}(\triangle)$ is a finite dimensional linear space. However, finding explicit formulae for its dimension for general $r, d$, and $\triangle$, is an extremely difficult problem due to the fact that the dimension depends not only on the way in which the

1) Department of Mathematics, University of Utah, 155 South 1400 East, JWB 233, Salt Lake City, Utah 84112-0090, pa@math.utah.edu.

2) Department of Mathematics, Vanderbilt University, Nashville, TN 37240, s@mars.cas.vanderbilt.edu. 
tetrahedra are connected to each other, but also on the precise location of the vertices. In general, an arbitrarily small change of the location of the vertices can change the dimension of $\mathcal{S}_{d}^{r}(\triangle)$.

The dimension of $\mathcal{S}_{d}^{r}(\triangle)$ can be trivially determined when $r=0$ or when $r=d$, see Remark 1. Our aim in this paper is to establish upper and lower bounds on the dimension of $\mathcal{S}_{d}^{r}(\triangle)$ for all choices of $0<r<d$, and for arbitrary tetrahedral partitions. Our bounds will depend on $d$ and $r$, and also on the nature of $\triangle$, but not on the precise locations of the vertices.

The paper is organized as follows. In Section 2 we describe how completely general tetrahedral partitions can be built inductively using 28 specific types of assembly steps. In Section 3 we show that lower and upper bounds on the dimension of $\mathcal{S}_{d}^{r}(\triangle)$ for arbitrary $0<r<d$ and arbitrary $\triangle$ can be given in terms of lower and upper bounds $\ell_{i}, u_{i}$ on how much the dimension of a spline space changes when we add just one tetrahedron using assembly step $i$ to an existing tetrahedral partition. Section 4 is devoted to a review of basic Bernstein-Bézier theory, while in the following section we present several formulae for counting certain sets of domain points. In Section 6 we derive explicit formulae for suitable $\ell_{i}$ and $u_{i}$ in terms of $r$ and $d$. In Section 7 we show that our lower and upper bounds take a strikingly simple form in the special case where the partition $\triangle$ is shellable. Several numerical examples illustrating our bounds are given in Section 8. We conclude the paper with remarks and references.

\section{$\S 2$. Tetrahedral Partitions}

Every tetrahedral partition $\triangle$ consisting of $N$ tetrahedra can be assembled in $N$ steps, starting with a single tetrahedron, and adding one additional tetrahedron in each subsequent step. Generally, there will be many different ways to assemble a given partition, and we will see later that the order in which the tetrahedra are added will affect our bounds on the dimension of the associated spline spaces.

For our purposes, we need to identify and classify the different ways in which a tetrahedron can be added to a partially completed partition in order to build a partition $\triangle$. There are twenty-eight types of assembly steps, which we number as in Tab. 1. For example, simply adding one tetrahedron that doesn't touch any existing tetrahedron at all is classified as type 0 . Adding a tetrahedron that touches the existing partition at one vertex only is classified as type 1 . A step of type 27 corresponds to adding a tetrahedron that touches the existing partition along exactly one face and also at the opposite vertex. The last column in the table is designed to help visualize these steps. In particular, assuming the tetrahedron being added is $T:=\left\langle v_{1}, v_{2}, v_{3}, v_{4}\right\rangle$, this column lists the vertices, edges, and faces of $T$ where $T$ touches the existing partition. Here $e_{i j}$ denotes the edge $\left\langle v_{i}, v_{j}\right\rangle$, and $f_{i j k}$ denotes the face $\left\langle v_{i}, v_{j}, v_{k}\right\rangle$.

These twenty-eight types of assembly steps allow the step-wise construction of completely general tetrahedral partitions. However, most partitions likely to be used in practice, including those with holes and cavities, can be built with only a few of these steps, see Remark 4. 


\begin{tabular}{|c|c|c|}
\hline Type & Nature of Touching Facets & List of Touching Facets \\
\hline 0 & Isolated tetrahedron & \\
\hline 1 & one vertex & $v_{1}$ \\
\hline 2 & two vertices & $v_{1}, v_{2}$, \\
\hline 3 & three vertices & $v_{1}, v_{2}, v_{3}$ \\
\hline 4 & four vertices & $v_{1}, v_{2}, v_{3}, v_{4}$ \\
\hline 5 & one edge & $e_{12}$ \\
\hline 6 & two opposite edges & $e_{12}, e_{34}$ \\
\hline 7 & two edges sharing a vertex & $e_{12}, e_{23}$ \\
\hline 8 & three edges forming a path & $e_{12}, e_{23}, e_{34}$ \\
\hline 9 & three edges sharing a vertex & $e_{12}, e_{13}, e_{14}$ \\
\hline 10 & three edges on one face & $e_{12}, e_{23}, e_{31}$ \\
\hline 11 & four edges forming a loop & $e_{12}, e_{23}, e_{34}, e_{41}$ \\
\hline 12 & four edges not forming a loop & $e_{12}, e_{23}, e_{31}, e_{14}$ \\
\hline 13 & five edges & $e_{12}, e_{13}, e_{14}, e_{23}, e_{24}$ \\
\hline 14 & six edges & $e_{12}, e_{13}, e_{14}, e_{23}, e_{24}, e_{34}$ \\
\hline 15 & one edge and one vertex & $e_{12}, v_{3}$ \\
\hline 16 & one edge and two vertices & $e_{12}, v_{3}, v_{4}$ \\
\hline 17 & two edges and one vertex & $e_{12}, e_{23}, v_{4}$ \\
\hline 18 & three edges and one vertex & $e_{12}, e_{23}, e_{31}, v_{4}$ \\
\hline 19 & one face and one edge & $f_{123}, e_{14}$ \\
\hline 20 & one face and two edges & $f_{123}, e_{14}, e_{24}$ \\
\hline 21 & one face and three edges & $f_{123}, e_{14}, e_{24}, e_{34}$ \\
\hline 22 & two faces and one edge & $f_{123}, f_{124}, e_{34}$ \\
\hline 23 & one face & $f_{123}$ \\
\hline 24 & two faces & $f_{123}, f_{124}$ \\
\hline 25 & three faces & $f_{123}, f_{124}, f_{134}$ \\
\hline 26 & four faces & $f_{123}, f_{124}, f_{134}, f_{234}$ \\
\hline 27 & one face and the opposite vertex & $f_{123}, v_{4}$ \\
\hline
\end{tabular}

Tab. 1. Assembly steps for tetrahedral partitions.

\section{$\S 3$. The Key Inequalities}

It is clear that when $\triangle$ consists of a single tetrahedron, the dimension of $\mathcal{S}_{d}^{r}(\triangle)$ is equal to the dimension of $\mathcal{P}_{d}$ which is $\left(\begin{array}{c}d+3 \\ 3\end{array}\right)$. To get bounds on the dimension of $\mathcal{S}_{d}^{r}(\triangle)$ on an arbitrary tetrahedral partition $\triangle$, we proceed by induction. Suppose $\triangle$ can be obtained from a tetrahedral partition $\tilde{\triangle}$ by adding one tetrahedron $T$. To get bounds on $\mathcal{S}_{d}^{r}(\triangle)$, we need to account for how the dimension changes as we add $T$ to $\tilde{\triangle}$. Clearly, this will depend on how $T$ joins $\tilde{\triangle}$, i.e., to which of the 28 classes identified in the previous section it belongs. For each $0 \leq i \leq 27$, let $\ell_{i}$ and $u_{i}$ be such that

$$
\ell_{i} \leq \operatorname{dim} \mathcal{S}_{d}^{r}(\triangle)-\operatorname{dim} \mathcal{S}_{d}^{r}(\tilde{\triangle}) \leq u_{i}
$$

whenever $T$ is of type $i$. We now establish the key inequalities. 
Theorem 3.1. Fix $0<r<d$, and suppose $\triangle$ is a tetrahedral partition that can be built inductively using $\tau_{i}$ assembly steps involving tetrahedra of type $i$ for $i=0, \ldots, 27$. Then

$$
L(r, d) \leq \operatorname{dim} \mathcal{S}_{d}^{r}(\triangle) \leq U(r, d),
$$

where

$$
L(r, d):=\max \left[\left(\begin{array}{c}
d+3 \\
3
\end{array}\right), \sum_{i=0}^{27} \tau_{i} \ell_{i}\right], \quad U(r, d):=\sum_{i=0}^{27} \tau_{i} u_{i} .
$$

Proof: Let $T_{1}, \ldots, T_{N}$ be a numbering of the tetrahedra in the order that they are added in building $\triangle$. The spline space $\mathcal{S}_{d}^{r}(\triangle)$ restricted to the single tetrahedron $T_{1}$ has dimension $\ell_{0}=u_{0}=\left(\begin{array}{c}d+3 \\ 3\end{array}\right)$. Now each time we add a tetrahedron of type $i$, the dimension changes by an amount $\delta_{i}$ satisfying $\ell_{i} \leq \delta_{i} \leq u_{i}$. Summing gives the formulae for $L(r, d)$ and $U(r, d)$, where for $L(r, d)$ we have taken the maximum with $\left(\begin{array}{c}d+3 \\ 3\end{array}\right)$ since $\mathcal{P}_{d} \subseteq \mathcal{S}_{d}^{r}(\triangle)$ for all $0 \leq r \leq d$.

Formulae for the $\ell_{i}$ and $u_{i}$ will be derived in Sect. 6 below. We refer to the $\tau_{i}$ as the assembly parameters of $\triangle$. In general they are not uniquely determined. A given tetrahedral partition can usually be built in many different ways, leading to different bounds. We give an example to illustrate this in Sect. 8, which also contains several other numerical examples illustrating Theorem 3.1.

\section{§4. Bernstein-Bézier Methods}

Our analysis of the dimension of $\mathcal{S}_{d}^{r}(\triangle)$ is based on well-known Bernstein-Bézier techniques, see [3] and references therein. For convenience, we review some of the basic ideas and notation. Suppose $T:=\left\langle v_{1}, v_{2}, v_{3}, v_{4}\right\rangle$ is a tetrahedron with vertices $v_{1}, v_{2}, v_{3}, v_{4}$. The key fact is that every polynomial $p \in \mathcal{P}_{d}$ has a unique Bernstein-Bézier representation of the form

$$
p:=\sum_{i+j+k+l=d} c_{i j k l} B_{i j k l},
$$

where $B_{i j k l}$ are the Bernstein basis polynomials of degree $d$ associated with $T$. It is standard practice to associate each coefficient $c_{i j k l}$ with a corresponding domain point

$$
\xi_{i j k l}:=\frac{i v_{1}+j v_{2}+k v_{3}+l v_{4}}{d},
$$

for $i+j+k+l=d$. Given $r \geq 0$, we often work with the following subsets of domain points:

- ball of radius $r$ around $v_{1}: B_{r}\left(v_{1}\right):=\left\{\xi_{i j k l}: i \geq d-r\right\}$,

- shell of radius $r$ around $v_{1}: R_{r}\left(v_{1}\right):=\left\{\xi_{i j k l}: i=d-r\right\}$,

- tube of radius $r$ around $e:=\left\langle v_{i}, v_{j}\right\rangle: t_{r}(e):=\left\{\xi_{i j k l}: i+j \geq d-r\right\}$,

- $r$-slab containing the face $F:=\left\langle v_{1}, v_{2}, v_{3}\right\rangle: G_{r}(F):=\left\{\xi_{i j k l}: l \leq r\right\}$. 
Analogous sets can be defined in the same way for the other vertices, edges, and faces.

One of the reasons that the Bernstein-Bézier representation is so useful is that it provides a simple way to describe when two polynomials defined on tetrahedra $T:=\left\langle v_{1}, v_{2}, v_{3}, v_{4}\right\rangle$ and $\widetilde{T}:=\left\langle v_{5}, v_{2}, v_{3}, v_{4}\right\rangle$ sharing the face $F:=\left\langle v_{2}, v_{3}, v_{4}\right\rangle$ join with $C^{r}$ smoothness. The condition is that

$$
\tilde{c}_{m i j k}=\sum_{\nu+\mu+\kappa+\delta=m} c_{\nu, i+\mu, j+\kappa, k+\delta} B_{\nu \mu \kappa \delta}^{m}\left(v_{5}\right), \quad \text { all } i+j+k=d-m
$$

for $m=0, \ldots, r$, where $\left\{c_{i j k l}\right\}_{i+j+k+l=d}$ and $\left\{\tilde{c}_{i j k l}\right\}_{i+j+k+l=d}$ are the B-coefficients of $p$ and $\tilde{p}$ relative to $T$ and $\widetilde{T}$, respectively.

We refer to the domain point $\tilde{\xi}_{m i j k}$ corresponding to the coefficient $\tilde{c}_{m i j k}$ on the left in (4.1) as the tip of the smoothness condition. It is clear from (4.1) that the conditions for two polynomials to join with $C^{r}$ smoothness across a common face involve only coefficients corresponding to domain points in the $r$-slabs containing $F$ in both $T$ and $\widetilde{T}$.

\section{$\S 5$. Formulae for Intersections of Balls, Tubes, and Slabs}

In this section we provide formulae for the sizes of various subsets of domain points. They are used in the following section to derive formulae for the expressions $\ell_{i}$ and $u_{i}$ appearing in Theorem 3.1. Given $0 \leq r<d$, let

- $n_{r}:=$ the number of points in $B_{r}(v)$, where $v$ is a vertex of $T$,

- $m_{2 b}(r, d):=$ the number of points in $B_{r}\left(v_{1}\right) \cap B_{r}\left(v_{2}\right)$, where $v_{1}, v_{2}$ are vertices of $T$,

- $m_{3 b}(r, d):=$ the number of points in $B_{r}\left(v_{1}\right) \cap B_{r}\left(v_{2}\right) \cap t_{r}\left(v_{3}\right)$, where $v_{1}, v_{2}, v_{3}$ are vertices of $T$,

- $m_{4 b}(r, d):=$ the number of points in $B_{r}\left(v_{1}\right) \cap B_{r}\left(v_{2}\right) \cap B_{r}\left(v_{3}\right) \cap B_{r}\left(v_{4}\right)$, where $v_{1}, v_{2}, v_{3}, v_{4}$ are the vertices of $T$,

- $m_{1 t}(r, d):=$ the number of points in $t_{r}(e)$, where $e$ is an edge of $T$,

- $m_{2 t}(r, d):=$ the number of points in $t_{r}\left(e_{1}\right) \cap t_{r}\left(e_{2}\right)$, where $e_{1}, e_{2}$ are edges of $T$ sharing a vertex $v$,

- $m_{t o}(r, d):=$ the number of points in $t_{r}\left(e_{1}\right) \cap t_{r}\left(e_{2}\right)$, where $e_{1}, e_{2}$ are edges of $T$ not sharing any vertex,

- $m_{3 t}(r, d):=$ the number of points in $t_{r}\left(e_{1}\right) \cap t_{r}\left(e_{2}\right) \cap t_{r}\left(e_{3}\right)$, where $e_{1}, e_{2}, e_{3}$ are edges of $T$ sharing a vertex $v$ of $T$,

- $m_{3 t f}(r, d):=$ the number of points in $t_{r}\left(e_{1}\right) \cap t_{r}\left(e_{2}\right) \cap t_{r}\left(e_{3}\right)$, where $e_{1}, e_{2}, e_{3}$ are edges of $T$ lying in one face,

- $m_{3 t p}(r, d):=$ the number of points in $t_{r}\left(e_{1}\right) \cap t_{r}\left(e_{2}\right) \cap t_{r}\left(e_{3}\right)$, where $e_{1}, e_{2}, e_{3}$ are edges of $T$ forming a path, i.e., not all lying on one face and not all sharing a single vertex, 
- $m_{4 t l}(r, d):=$ the number of points in $t_{r}\left(e_{1}\right) \cap t_{r}\left(e_{2}\right) \cap t_{r}\left(e_{3}\right) \cap t_{r}\left(e_{4}\right)$, where $e_{1}, \ldots, e_{4}$ are edges of $T$ forming a loop, i.e., such that no three of the edges share a single vertex,

- $m_{t b}(r, d):=$ the number of points in $t_{r}(e) \cap B_{r}(v)$, where $e$ is an edge of $T$ and $v$ is a vertex not contained in $e$,

- $m_{t 2 b}(r, d):=$ the number of points in $t_{r}(e) \cap B_{r}(v) \cap B_{r}(w)$, where $e$ is an edge of $T$ and $v, w$ are vertices not contained in $e$,

- $m_{2 t b}(r, d):=$ the number of points in $t_{r}\left(e_{1}\right) \cap t_{r}\left(e_{2}\right) \cap B_{r}(v)$, where $e_{1}, e_{2}$ are edges of $T$ and $v$ is a vertex not contained in either edge,

- $m_{t s}(r, d):=$ the number of points in $t_{r}(e) \cap G_{r}(F)$, where $e$ is an edge of $T$ not lying in the face $F$.

- $m_{2 t 2 b}(r, d):=$ the number of points in $\left(B_{r}\left(v_{1}\right) \cap B_{r}\left(v_{2}\right)\right) \backslash t_{r}(e)$, where $e$ is the edge with vertices $v_{3}$ and $v_{4}$,

- $m_{s}(r, d):=$ the number of points in the slab $G_{r}(F)$ associated with a face $F$ of $T$.

We now derive formulae for all of these expressions.

Lemma 5.1. For all $0 \leq r<d$,

$$
\begin{aligned}
n_{r} & :=\left(\begin{array}{c}
r+3 \\
3
\end{array}\right), \\
m_{2 b}(r, d) & :=\left(\begin{array}{c}
2 r-d+3 \\
3
\end{array}\right), \\
m_{3 b}(r, d) & :=\left(\begin{array}{c}
3 r-2 d+3 \\
3
\end{array}\right), \\
m_{4 b}(r, d) & :=\left(\begin{array}{c}
4 r-3 d+3 \\
3
\end{array}\right), \\
m_{1 t}(r, d) & :=\left(\begin{array}{c}
r+3 \\
3
\end{array}\right)+\sum_{\nu=r+1}^{d}\left(\begin{array}{c}
r+2 \\
2
\end{array}\right), \\
m_{2 t}(r, d) & :=\left(\begin{array}{c}
r+3 \\
3
\end{array}\right)+\sum_{\nu=r+1}^{d}\left(\begin{array}{c}
2 r+2-\nu \\
2
\end{array}\right), \\
m_{3 t}(r, d) & :=\left(\begin{array}{c}
r+3 \\
3
\end{array}\right)+\sum_{\nu=r+1}^{d}\left(\begin{array}{c}
3 r+2-2 \nu \\
2
\end{array}\right), \\
m_{t o}(r, d) & :=\left\{\begin{array}{c}
\sum_{\nu=d-r}^{r}(\nu+1)(d+1-\nu), \\
0,
\end{array} \quad \text { otherwise, } d \leq 2 r,\right.
\end{aligned}
$$




$$
\begin{aligned}
& m_{3 t f}(r, d):=\sum_{\nu=0}^{r}\left[\left(\begin{array}{c}
3 r-2 \nu-d+2 \\
2
\end{array}\right)-3\left(\begin{array}{c}
2 r-\nu-d+1 \\
2
\end{array}\right)\right] \\
& m_{3 t p}(r, d):=\sum_{\nu=d-r}^{r} \sum_{\mu=0}^{\nu}(d+1-\max (\nu, d-r+\mu)), \\
& m_{4 t l}(r, d):=\sum_{\nu=d-r}^{r} \sum_{\mu=0}^{\nu}(\min (d, r+\mu)+1-\max (\nu, d-r+\mu)) \text {, } \\
& m_{t b}(r, d):=\sum_{\nu=0}^{2 r-d}(\nu+1)(r-\nu+1) \\
& m_{t 2 b}(r, d):=\sum_{\nu=0}^{3 r-2 d}(\nu+1)(2 r-d-\nu+1) \\
& m_{2 t b}(r, d):=\sum_{\nu=0}^{2 r-d}\left[(\nu+1)^{2}-\left(\begin{array}{c}
r-d+\nu+1 \\
2
\end{array}\right)\right] \\
& m_{t s}(r, d):=\sum_{\nu=0}^{r}\left(\begin{array}{c}
\min (r, d-\nu)+2 \\
2
\end{array}\right) \\
& m_{2 t 2 b}(r, d):=\sum_{i=d-r}^{r} \sum_{j=\max (d-r, r+1-i)}^{d-i}(d-i-j+1), \\
& m_{s}(r, d):=\left(\begin{array}{c}
d+3 \\
3
\end{array}\right)-\left(\begin{array}{c}
d+2-r \\
3
\end{array}\right) .
\end{aligned}
$$

Proof: The formula for $n_{r}, m_{2 b}, m_{3 b}, m_{4 b}$ and $m_{s}$ can be obtained by inspection. We now consider the other cases individually.

$m_{1 t}$ : To get the formula for $m_{1 t}$, note that the tube contains the ball of radius $r$ around one of the vertices $v$ of $e$. In addition, for each $\nu=r+1, \ldots, d$, it contains $\left(\begin{array}{c}r+2 \\ 2\end{array}\right)$ points on the shell $R_{\nu}(v)$. Summing over the shells gives $m_{1 t}$.

$m_{2 t}$ : To get the formula for $m_{2 t}$, note that the ball of radius $r$ around $v$ is in both tubes, which accounts for the term $\left(\begin{array}{c}r+3 \\ 3\end{array}\right)$. To count the remaining points in the intersection of the two tubes, we consider the shells $R_{\nu}(v)$ for $\nu=r+1, \ldots, d$. It is easy to check that on the shell $R_{\nu}(v)$, there are $\left(\begin{array}{c}2 r+2-\nu \\ 2\end{array}\right)$ points in the intersection. Summing over the shells gives the second term in the formula for $m_{2 t}$.

$m_{3 t}$ : For the case of three intersecting tubes sharing the vertex $v$, we note that the number of points in the intersection lying on the shell $R_{\nu}(v)$ is $\left(\begin{array}{c}\nu+2-3(\nu-r) \\ 2\end{array}\right)$. Summing over the shells gives the formula for $m_{3 t}$. 
$m_{t o}$ : Without loss of generality we may assume the first tube is along the edge $e_{1}:=\left\langle v_{1}, v_{2}\right\rangle$ while the second is along the edge $e_{2}:=\left\langle v_{3}, v_{4}\right\rangle$. Then $\xi_{i j k l}$ lies in both tubes if and only if $i+j \geq d-r$ and $k+l \geq d-r$ which implies that $i+j \leq d-(d-r)=r$. Thus, for a point $\xi_{i j k l}$ to be in the intersection we need $d-r \leq i+j \leq r$. It follows that the intersection of the two tubes is empty if $d>2 r$. Letting $\nu=i+j$, there are $\nu+1$ ways to choose $i$ and $j$, and for each such choice, there are $d+1-\nu$ ways to choose $k$ and $l$ with $k+l=d-\nu$. The count follows.

$m_{3 t f}$ : Suppose the face is $F:=\left\langle v_{1}, v_{2}, v_{3}\right\rangle$. The expression in brackets is just the number of points in the intersection of the shell $R_{d-\nu}\left(v_{4}\right)$ and the tubes around the three edges of $F$. The result follows by summing over the shells.

$m_{3 t p}$ : For each $0 \leq i, j \leq 4$, let $t_{i j}$ be the tube along edge $e_{i j}:=\left\langle v_{i}, v_{j}\right\rangle$. We suppose the edges forming the path are $e_{12}, e_{23}, e_{34}$, and consider the three tubes $t_{12}, t_{23}$, and $t_{34}$. We need to count the number of 4 -tuples $(i, j, k, l)$ of nonnegative integers that satisfy

$$
i+j+k+l=d, \quad i+j \geq d-r, \quad j+k \geq d-r, \quad \text { and } \quad k+l \geq d-r .
$$

These inequalities imply that $i+j \leq r$ and $k+l \leq r$. It is convenient to use the substitution $\mu=i, \nu=i+j$, and $\kappa=i+j+k$. Then, according to the above inequalities, admissible values of $\nu$ range from $d-r$ to $r$, and admissible values of $\mu$ range from 0 to $\nu$. Since $j+k=\kappa-\mu \geq d-r$, we need $\kappa \geq d-r+\mu$. Given $\mu$ and $\nu$, there are thus $d+1-\max \{\nu, d-r+\mu\}$ choices of $\kappa$ ranging from $\max \{\nu, d-r+\mu\}$ to $d$. Counting the total number of possibilities gives the formula for $m_{3 t p}$.

$m_{4 t l}$ : The proof is similar to the proof for $m_{3 t p}$ except that now we also have to satisfy $\kappa-\mu=j+k \leq r$.

$m_{t b}$ : For each $d-r \leq \nu \leq r$, points in the intersection lie on $\nu-d+r+1$ lines parallel to $e$, each of which contains $d+1-\nu$ points. Thus, the total number is $\sum_{\nu=d-r}^{r}(\nu-d+r+1)(d+1-\nu)$. A change of summation index gives the stated formula.

$m_{t 2 b}$ : For each $2 d-2 r \leq \nu \leq r$, points in the intersection lie on $\nu-2 d+2 r+1$ lines parallel to $e$, each of which contains $d+1-\nu$ points. Thus, the total number is $\sum_{\nu=2 d-2 r}^{r}(\nu-2 d+2 r+1)(d+1-\nu)$. A change of summation index gives the stated formula.

$m_{2 t b}$ : Suppose the two edges are $e_{1}$ and $e_{2}$, and that the vertex not on the edges is $v_{4}$. For each $d-r \leq \ell \leq r$, the number of points in $t_{r}\left(e_{1}\right) \cap t_{r}\left(e_{2}\right) \cap R_{\ell}\left(v_{4}\right)$ is $(r-d+\ell+1)^{2}-\left(\begin{array}{c}2 r-2 \\ 2\end{array}\right)$. Summing over $\ell$ and changing the index gives the stated formula.

$m_{t s}$ : Let $v$ be the vertex opposite the slab. For each $0 \leq \nu \leq r$, the number of points in the intersection and lying on the shell $R_{d-\nu}(v)$ is $\left(\begin{array}{c}\min (r, d-\nu)+2 \\ 2\end{array}\right)$, and the formula follows. 
$m_{2 t 2 b}$ : A domain point $\xi_{i j k l}$ lies in the set $B_{r}\left(v_{1}\right) \cap B_{r}\left(v_{2}\right)$ if and only if $i \geq d-r$ and $j \geq d-r$. It lies outside the tube $t_{r}(e)$ if and only if $k+l<d-r$. We compute the number of 4 -tuples $(i, j, k, l)$ satisfying all three inequalities. The first two inequalities imply that $i, j \leq r$. The third inequality implies that $j \geq r+1-i$. Given $i$ and $j$ there are $d+1-i-j$ possible choices of $k$ and $l$. Summing gives the formula.

\section{$\S 6$. The Change in Dimension When Adding One Tetrahedron}

Suppose $\triangle$ is a tetrahedral partition that can be obtained from a tetrahedral partition $\tilde{\triangle}$ by adding one tetrahedron $T$. In this section we give upper and lower bounds $u_{i}$ and $\ell_{i}$ on the change in dimension

$$
\delta_{i}:=\operatorname{dim} \mathcal{S}_{d}^{r}(\triangle)-\operatorname{dim} \mathcal{S}_{d}^{r}(\tilde{\triangle})
$$

when $T$ is of type $i, 0 \leq i \leq 27$, according to the classification scheme of Sect. 3 . Note that $\delta_{i}$ can be negative in some cases.

Suppose $s \in \mathcal{S}_{d}^{r}(\triangle)$ and that we already know the coefficients of $s$ corresponding to domain points in $\tilde{\triangle}$. If $T$ touches $\tilde{\triangle}$ at a vertex $v$, then the $C^{r}$ smoothness at $v$ imposes $n_{r}$ conditions on the coefficients of $s$ associated with domain points in $B_{r}(v)$. Similarly, if $T$ touches $\tilde{\triangle}$ along an edge $e$, then the $C^{r}$ smoothness along $e$ imposes $m_{1 t}$ conditions on the coefficients in the tube $t_{r}(e)$. If $T$ touches $\triangle$ along a face $F$, then the $C^{r}$ smoothness across $F$ imposes $m_{s}$ conditions on the coefficients in the slab $G_{r}(F)$. Recall that $n_{d}=\left(\begin{array}{c}d+3 \\ 3\end{array}\right)$ is the number of coefficients of $\left.s\right|_{T}$.

To get a lower bound $\ell_{i}$ for $\delta_{i}$, we can take $n_{d}-n_{\text {cond }}$, where $n_{\text {cond }}$ is the number of conditions on $\left.s\right|_{T}$ imposed by the fact that $T$ joins with $\widetilde{\triangle}$ with $C^{r}$ smoothness. However, some of these conditions may be redundant in the sense that one or more of them are automatically satisfied whenever the others are satisfied. Removing as many redundant conditions as possible leads to a better lower bound. There are two situations where we know that conditions on a coefficient $c_{\xi}$ are redundant:

1) $\xi \in B_{r}(v)$, where $v$ is contained in more than one of the edges or faces where $T$ touches $\triangle$. There is one smoothness condition associated with each such edge or face, but we need only count one of them as the rest are redundant.

2) $\xi \in t_{r}(e)$, where $e$ is contained in more than one of the faces where $T$ touches $\widetilde{\triangle}$. There is one smoothness condition associated with each such face, but we need only count one of them as the rest are redundant.

Note that $\ell_{i}$ computed in this way can be negative as well as nonnegative.

The change in dimension $\delta_{i}$ is equal to the number of coefficients of $\left.s\right|_{T}$ that can be set to arbitrary values while maintaining all smoothness conditions. Such coefficients cannot correspond to domain points which are tips of any smoothness condition. We call such points constrained points. Thus, as an upper bound we can take $u_{i}=n_{d}-n_{\text {tips }}$, where $n_{\text {tips }}$ is the number of points in $T$ that are constrained. While counting unconstrained points seems like a straightforward process, it is 
complicated in some cases due to the fact that balls, tubes, and slabs can intersect in various ways depending on $r, d$ and the class to which $T$ belongs. Note that $u_{i}$ computed in this way is nonnegative for all $0 \leq i \leq 27$.

In terms of the notation of the previous section, the number of domain points in a ball of radius $r$ around a vertex is $n_{r}$. For ease of notation we write $m_{1 t}:=$ $m_{1 t}(r, d)$, with a similar short-hand notation for all the other quantities defined in the previous section. For $d>2 r$, we define $\tilde{m}_{1 t}:=m_{1 t}(r, d-r-1), \tilde{m}_{2 t}:=m_{2 t}(r, d-$ $r-1), \tilde{m}_{3 t}:=m_{3 t}(r, d-r-1)$. Let $\hat{m}_{1 t}:=m_{1 t}(2 r-d, r), \hat{m}_{2 t}:=m_{2 t}(2 r-d, r)$, and $\hat{m}_{3 t f}:=m_{3 t f}(2 r-d, r)$ for $d \leq 2 r$, and define them to be zero when $d>2 r$. Given an edge $e:=\langle u, v\rangle$ of a tetrahedron, we define a 1-partial tube around $e$ to be $t_{r}(e) \backslash B_{r}(u)$. Similarly, we define a 2-partial tube to be $t_{r}(e) \backslash\left(B_{r}(u) \cup B_{r}(v)\right)$.

Lemma 6.1. The expressions given in Tab. 2 provide upper and lower bounds for $\delta_{i}$ for all $0 \leq i \leq 27$.

Proof: The cases 0, 1, 5, 23, and 27 are obvious.

2: $\left(T\right.$ touches $\tilde{\triangle}$ at two vertices $\left.v_{1}, v_{2}\right)$. There are $n_{r}$ smoothness conditions associated with each of the balls $B_{r}\left(v_{i}\right), i=1,2$, and the lower bound follows. The number of constrained points in $T$ is $2 n_{r}-m_{2 b}$, since points in the intersection of two balls should be counted only once. This gives the upper bound.

3: $\left(T\right.$ touches $\tilde{\triangle}$ at three vertices $\left.v_{1}, v_{2}, v_{3}\right)$. There are $n_{r}$ smoothness conditions associated with each of the balls $B_{r}\left(v_{i}\right), i=1,2,3$, and the lower bound follows. The number of points in the union of the three balls is $3 n_{r}-3 m_{2 b}+m_{3 b}$, and the upper bound follows.

4: $\left(T\right.$ touches $\tilde{\triangle}$ at four vertices $\left.v_{1}, v_{2}, v_{3}, v_{4}\right)$. There are $n_{r}$ smoothness conditions associated with each of the balls $B_{r}\left(v_{i}\right), i=1, \ldots, 4$, and the lower bound follows. The number of points in the union of the four balls is $4 n_{r}-6 m_{2 b}+4 m_{3 b}+m_{4 b}$, and the upper bound follows.

6: ( $T$ touches $\tilde{\triangle}$ along two edges $e_{1}, e_{2}$ that do not share a vertex). There are $m_{1 t}$ smoothness conditions associated with each of these edges, and the lower bound follows. The number of points in $t_{r}\left(e_{1}\right) \cup t_{r}\left(e_{2}\right)$ is $2 m_{1 t}-m_{t o}$, where the term $m_{t o}$ takes account of the fact that the tubes can intersect when $d \leq 2 r$. This gives the upper bound.

7: $\left(T\right.$ touches $\tilde{\triangle}$ along two edges $e_{1}$ and $e_{2}$ that share a vertex $\left.v\right)$. There are $m_{1 t}$ smoothness conditions associated with each of these edges. However, since both tubes contain $B_{r}(v), n_{r}$ of these conditions are redundant, and the lower bound follows. The number of points in $t_{r}\left(e_{1}\right) \cup t_{r}\left(e_{2}\right)$ is $2 m_{1 t}-m_{2 t}$, and the upper bound follows.

8: ( $T$ touches $\tilde{\triangle}$ along three edges $e_{1}, e_{2}, e_{3}$ forming a path). There are $m_{1 t}$ smoothness conditions associated with each of these edges. However, since the first two tubes $t_{r}(e)$ both contain $B_{r}\left(v_{1}\right), n_{r}$ of the conditions with tips in $B_{r}\left(v_{1}\right)$ are redundant. Similarly, $n_{r}$ of the conditions with tips in $B_{r}\left(v_{2}\right)$ are redundant, and the 


\begin{tabular}{|c|c|c|}
\hline$i$ & $\ell_{i}$ & $u_{i}$ \\
\hline 0 & $n_{d}$ & $n_{d}$ \\
\hline 1 & $n_{d}-n_{r}$ & $n_{d}-n_{r}$ \\
\hline 2 & $n_{d}-2 n_{r}$ & $n_{d}-2 n_{r}+m_{2 b}$ \\
\hline 3 & $n_{d}-3 n_{r}$ & $n_{d}-3 n_{r}+3 m_{2 b}-m_{3 b}$ \\
\hline 4 & $n_{d}-4 n_{r}$ & $n_{d}-4 n_{r}+6 m_{2 b}-4 m_{3 b}+m_{4 b}$ \\
\hline 5 & $n_{d}-m_{1 t}$ & $n_{d}-m_{1 t}$ \\
\hline 6 & $n_{d}-2 m_{1 t}$ & $n_{d}-2 m_{1 t}+m_{t o}$ \\
\hline 7 & $n_{d}-2 m_{1 t}+n_{r}$ & $n_{d}-2 m_{1 t}+m_{2 t}$ \\
\hline 8 & $n_{d}-3 m_{1 t}+2 n_{r}$ & $n_{d}-3 m_{1 t}+2 m_{2 t}+m_{t o}-m_{3 t p}$ \\
\hline 9 & $n_{d}-3 m_{1 t}+2 n_{r}$ & $n_{d}-3 m_{1 t}+3 m_{2 t}-m_{3 t}$ \\
\hline 10 & $n_{d}-3 m_{1 t}+3 n_{r}-m_{3 b}$ & $n_{d}-3 m_{1 t}+3 m_{2 t}-m_{3 t f}$ \\
\hline 11 & $n_{d}-4 m_{1 t}+4 n_{r}-m_{4 b}$ & $n_{d}-4 m_{1 t}+4 m_{2 t}+2 m_{t o}-4 m_{3 t p}+m_{4 t l}$ \\
\hline 12 & $n_{d}-4 m_{1 t}+4 n_{r}-m_{3 b}$ & $\begin{cases}0, & \text { if } d \leq 2 r \\
n_{d}-4 m_{1 t}+5 m_{2 t}-m_{3 t}-m_{3 t f}, & \text { if } d>2 r\end{cases}$ \\
\hline 13 & $n_{d}-5 m_{1 t}+6 n_{r}-2 m_{3 b}$ & $\begin{cases}0, & \text { if } d \leq 2 r \\
n_{d}-5 m_{1 t}+8 m_{2 t}-2 m_{3 t f}-2 m_{3 t}, & \text { if } d>2 r\end{cases}$ \\
\hline 14 & $n_{d}-6 m_{1 t}+8 n_{r}-4 m_{3 b}+m_{4 b}$ & $\begin{cases}0, & \text { if } d \leq 2 r \\
n_{d}-6 m_{1 t}+12 m_{2 t}-4 m_{3 t}-4 m_{3 t f}, & \text { if } d>2 r\end{cases}$ \\
\hline 15 & $n_{d}-m_{1 t}-n_{r}$ & $n_{d}-m_{1 t}-n_{r}+m_{t b}$ \\
\hline 16 & $n_{d}-m_{1 t}-2 n_{r}$ & $n_{d}-m_{1 t}-2 n_{r}+2 m_{t b}+m_{2 b}-m_{t 2 b}$ \\
\hline 17 & $n_{d}-2 m_{1 t}$ & $n_{d}-2 m_{1 t}-n_{r}+m_{2 t}+2 m_{t b}-m_{2 t b}$ \\
\hline 18 & $n_{d}-3 m_{1 t}+2 n_{r}-m_{3 b}$ & $\begin{array}{c}n_{d}-n_{r}-3\left(m_{1 t}-\hat{m}_{1 t}-m_{2 t}+\hat{m}_{2 t}\right) \\
-\left(m_{3 t f}-\hat{m}_{3 t f}\right)\end{array}$ \\
\hline 19 & $n_{d}-m_{s}-m_{1 t}+n_{r}$ & $n_{d}-m_{s}-m_{1 t}+m_{t s}$ \\
\hline 20 & $n_{d}-m_{s}-2 m_{1 t}+3 n_{r}-m_{3 b}$ & $\begin{cases}0, & \text { if } d \leq 2 r \\
n_{d}-m_{s}-2 \tilde{m}_{1 t}+\tilde{m}_{2 t}, & \text { if } d>2 r\end{cases}$ \\
\hline 21 & $n_{d}-m_{s}-3 m_{1 t}+5 n_{r}-3 m_{3 b}+m_{4 b}$ & $\begin{cases}0, & \text { if } d \leq 2 r \\
n_{d}-m_{s}-3 \tilde{m}_{1 t}+3 \tilde{m}_{2 t}-\tilde{m}_{3 t}, & \text { if } d>2 r\end{cases}$ \\
\hline 22 & $n_{d}-2 m_{s}+2 n_{r}-m_{2 b}+m_{2 t 2 b}$ & $\begin{cases}0, & \text { if } d \leq 3 r \\
n_{d-2 r-2}-m_{1 t}+2 m_{t s}, & \text { if } d>3 r\end{cases}$ \\
\hline 23 & $n_{d}-m_{s}$ & $n_{d-r-1}$ \\
\hline 24 & $n_{d}-2 m_{s}+m_{1 t}$ & $n_{d-2 r-2}$ \\
\hline 25 & $n_{d}-3 m_{s}+3 m_{1 t}-m_{3 t}$ & $n_{d-3 r-3}$ \\
\hline 26 & $\begin{cases}-4\left(m_{s}-3 m_{1 t}+3 m_{2 t}-m_{3 t f}\right), & \text { if } d \leq 2 r \\
n_{d}-4 m_{s}+6 m_{1 t}-4 m_{3 t}, & \text { if } d>2 r\end{cases}$ & $n_{d-4 r-4}$ \\
\hline 27 & $n_{d}-m_{s}-n_{r}$ & $\left(n_{d}-m_{s}-n_{r}\right)_{+}$ \\
\hline
\end{tabular}

Tab. 2. Formulae for $\ell_{i}$ and $u_{i}$.

lower bound follows. The upper bound follows from the fact that the number of points in $t_{r}\left(e_{1}\right) \cup t_{r}\left(e_{2}\right) \cup t_{r}\left(e_{3}\right)$ is $3 m_{1 t}-2 m_{2 t}-m_{t o}+m_{3 t p}$, as can be seen by a careful examination of a Venn diagram.

9: $\left(T\right.$ touches $\tilde{\triangle}$ along three edges $e_{1}, e_{2}, e_{3}$ sharing a vertex $v$ ). There are $m_{1 t}$ smoothness conditions associated with each of these edges. However, since all three tubes contain $B_{r}(v), 2 n_{r}$ of these conditions are redundant, and the lower bound follows. The upper bound follows from the fact that the number of points in the intersection of all three tubes is $3 m_{1 t}-3 m_{2 t}+m_{3 t}$. 
10: ( $T$ touches $\tilde{\triangle}$ along three edges $e_{1}, e_{2}, e_{3}$ on one face). The three 2-partial tubes contain $3\left(m_{1 t}-2 n_{r}+m_{2 b}\right)$ points. The three balls contain $3 n_{r}-3 m_{2 b}+m_{3 b}$ points, cf. the argument for the upper bound in case 3 . Thus, there are at most $3 m_{1 t}-3 n_{r}+$ $m_{3 b}$, nonredundant smoothness conditions, and the lower bound follows. The upper bound follows from the fact that the number of points in $t_{r}\left(e_{1}\right) \cup t_{r}\left(e_{2}\right) \cup t_{r}\left(e_{3}\right)$ is $3 m_{1 t}-3 m_{2 t}+m_{3 t f}$.

11: ( $T$ touches $\tilde{\triangle}$ along four edges $e_{1}, \ldots, e_{4}$ forming a loop). Given a domain point $\xi$ in $T$, let $b(\xi)$ and $t(\xi)$ be the number of balls $B_{r}\left(v_{i}\right)$ and tubes $t_{r}\left(e_{i}\right)$, $1 \leq i \leq 4$, that contain $\xi$. Suppose $\xi$ is not in the intersection of all four balls. Then the coefficient associated with the point $\xi$ is subject to $t(\xi)$ conditions, but $b(\xi)$ of them are redundant, and thus the change in dimension corresponding to this point is bounded below by $1-t(\xi)+b(\xi)$. Points that are in all four balls are also in all four tubes. The associated coefficients are subject to four conditions, but three of them are redundant, and thus there is no change in dimension. It follows that a lower bound for the change in dimension when we add $T$ to $\tilde{\triangle}$ is given by $\sum_{\xi \in T}(1-t(\xi)+b(\xi))-m_{4 b}=n_{d}-4 m_{1 t}+4 n_{r}-m_{4 b}$. To get the upper bound, we need to count the number of points in $\Gamma:=\cup_{i=1}^{4} t_{r}\left(e_{i}\right)$. Looking at a Venn diagram, we see that there are four pairs of neighboring tubes intersecting in $m_{2 t}$ points and two pairs of opposite tubes that intersect in $m_{t o}$ points. There are four paths of three tubes, and $m_{3 t p}$ points in the intersection of the three tubes in such a path. The intersection of all four tubes contains $m_{4 t l}$ points. It follows that the number of points in $\Gamma$ is $4 m_{1 t}-4 m_{2 t}-2 m_{t o}+4 m_{3 t f}-m_{4 t l}$, and the upper bound follows.

12: ( $T$ touches $\tilde{\triangle}$ along four edges $e_{1}, \ldots, e_{4}$ not forming a loop). Three of these edges lie on one face $F$. Let $v_{1}, v_{2}, v_{3}$ be the vertices on this face, and suppose $v_{3}$ is the vertex where three of the touching edges meet. There are $m_{1 t}-2 n_{r}+m_{2 b}$ points in each of the three 2-partial tubes along the edges of $F$. In addition, there are $m_{1 t}-n_{r}$ points in the 1-partial tube along the fourth edge. There are $3 n_{r}-3 m_{2 b}+m_{3 b}$ points in the three balls around the vertices of $F$, cf. the argument for the upper bound in case 3 . Thus, the total number of nonredundant smoothness conditions is at most $4 m_{1 t}-4 n_{r}+m_{3 b}$, and the lower bound follows. To get the upper bound, we count the number of points in $\Gamma:=\cup_{i=1}^{4} t_{r}\left(e_{i}\right)$. It is easy to see that when $d \leq 2 r, \Gamma$ contains all points in $T$, and so $u_{12}=0$. Suppose now $d>2 r$. In this case $m_{3 t p}=m_{t o}=0$. Looking at a Venn diagram, we see there are five pairs of neighboring tubes intersecting in $m_{2 t}$ points, and one pair of opposite tubes intersecting in $m_{t o}$ points. There is one set of three tubes intersecting in $m_{3 t f}$ points, one set of three tubes meeting at $v_{3}$ intersecting in $m_{3 t}$ points, and 2 pairs of three tubes forming paths such that each pair intersects in $m_{3 t p}$ points. The intersection of all four tubes is empty. It follows that the number of points in $\Gamma$ is $4 m_{1 t}-5 m_{2 t}+m_{3 t}+m_{3 t f}$. This gives the upper bound.

13: $\left(T\right.$ touches $\widetilde{\triangle}$ along five edges $\left.e_{1}, \ldots, e_{5}\right)$. Let $v_{1}, v_{2}$ be the vertices where three of these edges meet, and $e_{34}$ the single non-touching edge. Then two of the touching edges meet at $v_{3}$, and two meet at $v_{4}$. Each of the five 2-partial tubes contains 
$m_{1 t}-2 n_{r}+m_{2 b}$ points. As discussed in case $3, B_{r}\left(v_{1}\right) \cup B_{r}\left(v_{2}\right) \cup B_{r}\left(v_{3}\right)$ contains $3 n_{r}-3 m_{2 b}+m_{3 b}$ points. There are $n_{r}-2 m_{2 b}+m_{3 b}$ points in $B_{r}\left(v_{4}\right)$ but not in the balls $B_{r}\left(v_{1}\right)$ or $B_{r}\left(v_{2}\right)$. We conclude that the total number of nonredundant smoothness conditions is at most $5 m_{1 t}-6 n_{r}+2 m_{3 b}$, and the lower bound follows. To get the upper bound, we count the number of points in $\Gamma:=\cup_{i=1}^{5} t_{r}\left(e_{i}\right)$. It is easy to see that $\Gamma$ contains all the points of $T$ when $d \leq 2 r$, and so $u_{13}=0$. Suppose now $d>2 r$. In this case $m_{3 t p}=m_{t o}=0$. Looking at a Venn diagram, we see that there are eight pairs of neighboring tubes intersecting in $m_{2 t}$ points, and two pairs of opposite tubes intersecting in $m_{t o}=0$ points. There are 2 sets of three tubes intersecting in $m_{3 t f}$ points and 2 sets of three tubes intersecting in $m_{3 t}$ points. The remaining 6 intersecting sets of triples of three tubes each contain $m_{3 t p}=0$ points. There are 6 sets where four tubes intersect, but the intersections are all empty since opposite tubes don't overlap. It follows that the number of constrained points is $5 m_{1 t}-8 m_{2 t}+2 m_{3 t f}+2 m_{3 t}$. This gives the upper bound.

14: ( $T$ touches $\tilde{\triangle}$ along six edges). There are $m_{1 t}-2 n_{r}+m_{2 b}$ points in each of the six 2-partial tubes around the edges. In addition, there are $4 n_{r}-6 m_{2 b}+4 m_{3 b}-m_{4 b}$ points in the four balls, cf. the discussion of the upper bound in case 4 . Thus, the total number of nonredundant smoothness conditions is at most $6 m_{1 t}-8 n_{r}+$ $4 m_{3 b}-m_{4 b}$, and the lower bound follows. To get the upper bound, we count the number of points in the union $\Gamma$ of the tubes around the six edges. It is easy to see that $\Gamma$ contains all the points of $T$ when $d \leq 2 r$, and so $u_{14}=0$. Suppose now $d>2 r$. In this case $m_{3 t p}=m_{t o}=0$. There are 15 pairs of intersecting tubes. Twelve of these intersect in $m_{2 t}$ points, and the other three intersect in $m_{t o}$ points. There are 20 sets of intersections of three tubes. Four of these contain $m_{3 t f}$ points, four of them contain $m_{3 t}$ points, and the remaining 12 contain $m_{3 t p}=0$ points. There are 6 sets where four tubes intersect, but the intersections are all empty since opposite tubes don't overlap. It follows that the number of points in $\Gamma$ is $6 m_{1 t}-12 m_{2 t}+4 m_{3 t}+4 m_{3 t f}$. This gives the upper bound.

15: ( $T$ touches $\tilde{\triangle}$ on one edge $e$ and at a vertex $v$ not on $e$ ). Clearly the number of nonredundant smoothness conditions is at most $m_{1 t}+n_{r}$, and the lower bound follows. The upper bound follows from the fact that there are $m_{1 t}+n_{r}-m_{t b}$ points in $t_{r}(e) \cup B_{r}(v)$.

16: ( $T$ touches $\tilde{\triangle}$ on one edge $e$ and at two vertices $v_{1}, v_{2}$ not on $e$ ). The number of smoothness conditions with tips in $T$ is $m_{1 t}+2 n_{r}$, and the lower bound follows. Examining a Venn diagram, we see that there are $m_{1 t}+2 n_{r}-2 m_{t b}-m_{2 b}+m_{t 2 b}$ points in $t_{r}(e) \cup B_{r}\left(v_{1}\right) \cup B_{r}\left(v_{2}\right)$, and the upper bound follows.

17: ( $T$ touches $\tilde{\triangle}$ on two edges $e_{1}, e_{2}$ and at a vertex $v$ not on those edges). Clearly the number of nonredundant smoothness conditions is at most $2 m_{1 t}-n_{r}+n_{r}=$ $2 m_{1 t}$, and the lower bound follows. The upper bound follows from the fact that there are $2 m_{1 t}+n_{r}-2 m_{t b}-m_{2 t}+m_{2 t b}$ points in $t_{r}\left(e_{1}\right) \cup t_{r}\left(e_{2}\right) \cup B_{r}(v)$.

18: ( $T$ touches $\tilde{\triangle}$ on three edges $e_{1}, e_{2}, e_{3}$ and at a vertex $v$ not on those edges). The number of nonredundant smoothness conditions associated with the three tubes is 
at most $3 m_{1 t}-3 n_{r}+m_{3 b}$, cf. the discussion of case 10. Adding the $n_{r}$ conditions associated with $B_{r}(v)$ leads to the lower bound. To get the upper bound we first notice that are $3 m_{1 t}-3 m_{2 t}+m_{3 t f}$ points in $\Lambda:=t_{r}\left(e_{1}\right) \cup t_{r}\left(e_{2}\right) \cup t_{r}\left(e_{3}\right)$, cf. the upper bound in case 10. There are $n_{r}$ points in the ball $B_{r}(v)$. However, if $d \leq 2 r$, the ball $B_{r}(v)$ intersects $\Lambda$ creating three smaller tubes of radius $2 r-d$. The number of points in these tubes is $3 \hat{m}_{1 t}-3 \hat{m}_{2 t}+\hat{m}_{3 t f}$, and the upper bound follows.

19: ( $T$ touches $\tilde{\triangle}$ on one face $F$ and one edge $e$ not in the face). The number of nonredundant smoothness conditions is at most $m_{s}+m_{1 t}-n_{r}$, and the lower bound follows. To get the upper bound, we observe that the number of points in $G_{r}(F) \cup t_{r}(e)$ is $m_{s}+m_{1 t}-m_{t s}$.

20: ( $T$ touches $\tilde{\triangle}$ on one face $F$ and two edges $e_{1}, e_{2}$ not in the face). Let $e_{3}$ be the edge lying on the same face as $e_{1}$ and $e_{2}$. The number of nonredundant smoothness conditions in the three tubes surrounding the edges $e_{1}, e_{2}, e_{3}$ is $3 m_{1 t}-3 n_{r}+m_{3 b}$, cf. the lower bound for case 10. The number of nonredundant smoothness conditions in the slab $G_{r}(F)$ but not in the tube $t_{r}\left(e_{3}\right)$ is $m_{s}-m_{1 t}$. Thus, the total number of nonredundant smoothness conditions is at most $m_{s}+2 m_{1 t}-3 n_{r}+m_{3 b}$, and the lower bound follows. Let $\Gamma$ be the union of the slab $G_{r}(F)$ with the tubes around the two edges $e_{1}, e_{2}$. It is easy to see that $\Gamma$ contains all points of $T$ when $d \leq 2 r$, and so $u_{20}=0$ in this case. Now suppose $d>2 r$. In this case there are $m_{s}$ constrained points in the slab $G_{r}(F)$. The set of domain points resulting from cutting off the slab can be considered as corresponding to a polynomial of degree $d-r-1$. Referring to case 7 , the number of points in the two tubes sharing the vertex $v_{4}$ is $2 \tilde{m}_{1 t}-\tilde{m}_{2 t}$. The upper bound follows.

21: ( $T$ touches $\tilde{\triangle}$ on one face $F$ and three edges $e_{1}, e_{2}, e_{3}$ not in the face). To count nonredundant smoothness conditions, we note that $T$ touches on all six edges. By case 14, the number of nonredundant smoothness conditions associated with these edges is at most $6 m_{1 t}-8 n_{r}+4 m_{3 b}-m_{4 b}$. The number of nonredundant smoothness conditions associated with points in the slab $G_{r}(F)$ but not in the tubes around the three edges of $F$ is $m_{s}-\left(3 m_{1 t}-3 n_{r}+m_{3 b}\right)$. Thus the total number of nonredundant smoothness conditions is at most $m_{s}+3 m_{1 t}-5 n_{r}+3 m_{3 b}-m_{4 b}$, and the lower bound follows. Let $\Gamma:=G_{r}(F) \cup t_{r}\left(e_{1}\right) \cup t_{r}\left(e_{2}\right) \cup t_{r}\left(e_{3}\right)$. It is easy to see that $\Gamma$ contains all points of $T$ when $d \leq 2 r$, and so $u_{21}=0$ in this case. Suppose now $d>2 r$. Then the number of points in the slab $G_{r}(F)$ is $m_{s}$, while the number of points in the rest of $\Gamma$ is $3 \tilde{m}_{1 t}-3 \tilde{m}_{2 t}+\tilde{m}_{3 t}$, cf. case 9 . This leads immediately to the upper bound.

22: ( $T$ touches $\tilde{\triangle}$ on two faces $F_{1}, F_{2}$ and one edge $e$ not in either face). If $d>2 r$, then the number of nonredundant conditions with tips in the two slabs is $2 m_{s}-m_{1 t}$, while the number of nonredundant conditions with tips in the tube $t_{r}(e)$ is at most $m_{1 t}-2 n_{r}+m_{2 b}$. The lower bound follows since $m_{2 t 2 b}=0$ in this case. Now suppose $d \leq 2 r$ and that $e:=\left\langle v_{3}, v_{4}\right\rangle$ is the edge between the two faces. In this case we have counted each point $\xi$ in $B_{r}\left(v_{1}\right) \cap B_{r}\left(v_{2}\right) \backslash t_{r}(e)$ twice, but the two smoothness conditions with tip at $\xi$ are are consistent because they are associated with the 
tube $t_{r}(e)$. Thus in this case we have to adjust the lower bound by adding $m_{2 t 2 b}$. It is easy to check that the upper bound is zero whenever $d \leq 3 r$. Suppose now $d>3 r$. Let $\widetilde{T}$ be the tetrahedron obtained by stripping off two slabs of size $r$ from $T$. Clearly, $\widetilde{T}$ contains $n_{d-2 r-2}$ points. The number of points lying in the tube of radius $r$ around the edge $e \cap \widetilde{T}$ is $m_{1 t}-2 m_{t s}$, and the upper bound follows.

24: ( $T$ touches $\tilde{\triangle}$ on two faces). The number of nonredundant smoothness conditions in the slabs associated with the two faces is $2 m_{s}-m_{1 t}$, and the lower bound follows. Since all points in these two slabs are constrained, it is clear that the number of unconstrained points is equal to the number of points in the tetrahedron that is obtained by slicing off the two slabs, namely $n_{d-2 r-2}$.

25: ( $T$ touches $\tilde{\triangle}$ on three faces $\left.F_{1}, F_{2}, F_{3}\right)$. Let $v$ be the vertex opposite the nontouching face, and let $e_{1}, e_{2}, e_{3}$ be the three edges meeting at $v$. The number of points contained in $T$ but not in the tubes around these edges is $u_{9}=n_{d}-3 m_{1 t}+$ $3 m_{2 t}-m_{3 t}$. The number of points in one of the three slabs $G_{r}\left(F_{i}\right)$ but not in the tubes is $m_{s}-2 m_{1 t}+m_{2 t}$. Multiplying the latter by three and subtracting gives the lower bound. The upper bound follows as in the previous case by removing three slabs.

26: ( $T$ touches $\tilde{\triangle}$ on four faces). Consider first the case where $d \leq 2 r$. In this case the number of constrained points is $n_{d}$, and so the upper bound is 0 . To get the lower bound, we subtract the number of smoothness conditions with tips outside the tubes around edges of $T$ from the number of unconstrained points (which is 0 ). For each face, the number of smoothness conditions outside the three tubes around the edges of that face is $m_{s}-\left(3 m_{1 t}-3 m_{2 t}+m_{3 t f}\right)$, cf. case 9 , and the lower bound follows. Now suppose $d>2 r$. In this case the number of points contained in $T$ but not in the six tubes is $u_{14}=n_{d}-6 m_{1 t}+12 m_{2 t}-4 m_{3 t}-4 m_{3 t f}$. The number of points in each of the four slabs but not in the tubes is $m_{s}-3 m_{1 t}+3 m_{2 t}-m_{3 t f}$. Multiplying the latter by four and subtracting gives the lower bound. To get the upper bound we strip off four slabs.

\section{$\S 7$. The Case Where $\triangle$ Is Shellable}

A tetrahedral partition $\triangle$ is called shellable provided it consists of a single tetrahedron, or can be obtained from a shellable tetrahedral partition $\tilde{\triangle}$ by adding one tetrahedron $T$ such that $T$ intersects $\tilde{\triangle}$ precisely along one, two, or three triangular faces. In this section we show that if $\triangle$ is shellable, then there are simple formulae for the bounds on $\operatorname{dim} \mathcal{S}_{d}^{r}(\triangle)$ in terms of the combinatorics of $\triangle$. Let $F_{I}, E_{I}, V_{I}$ be the number of interior faces, edges, and vertices of $\triangle$. Given $0 \leq r<d$, let $\beta:=m_{1 t}-n_{d}$ and $\gamma:=n_{d}-m_{3 t}$. Similarly, let $\tilde{\beta}:=n_{d-2 r-2}-2 n_{d-r-1}$ and $\tilde{\gamma}:=3 n_{d-r-1}-3 n_{d-2 r-2}+n_{d-3 r-3}$.

Theorem 7.1. Suppose $\triangle$ is a shellable tetrahedral partition. Then for every $0 \leq r<d$,

$$
L(r, d) \leq \operatorname{dim} \mathcal{S}_{d}^{r}(\triangle) \leq U(r, d)
$$


where

$$
\begin{aligned}
& L(r, d)=n_{d}+\left(n_{d-r-1} F_{I}+\beta E_{I}+\gamma V_{I}\right)_{+}, \\
& U(r, d)=n_{d}+n_{d-r-1} F_{I}+\tilde{\beta} E_{I}+\tilde{\gamma} V_{I} .
\end{aligned}
$$

Proof: If $\triangle$ is shellable, then it can be assembled using only steps of types 0,23 , 24 , and 25. Clearly, $\tau_{0}=1$. We get a new interior vertex each time we carry out step 25, and thus $V_{I}=\tau_{25}$. We get new interior edges each time we carry out steps of types 24 and 25 , and so $E_{I}=\tau_{24}+3 \tau_{25}$. Similarly, $F_{I}=\tau_{23}+2 \tau_{24}+3 \tau_{25}$. Solving these equations for the assembly parameters, we find that $\tau_{23}=F_{I}-2 E_{I}+3 V_{I}$, $\tau_{24}=E_{I}-3 V_{I}$, and $\tau_{25}=V_{I}$. Referring to Tab. 2 and using the fact that $n_{d}-m_{s}=$ $n_{d-r-1}$, the formulae (7.1) follows immediately from Theorem 3.1.

\section{$\S 8$. Numerical Examples}

We have extensively tested and evaluated our bounds using a java program which is capable of computing the exact dimension, see Remark 6 . In this section we describe the results of a few of our tests. Our first example shows that (at least for a range of $r$ and $d$ ), our lower bounds cannot be improved.

Example 8.1. For each $0 \leq i \leq 27$, let $\triangle_{i}$ be the smallest tetrahedral partition such that to build $\triangle_{i}$ we use an assembly step of type $i$ as the last step.

Discussion: The $\triangle_{i}$ are actually quite small. For example, for $i=1, \ldots, 4, \triangle_{i}$ consists of just $i+1$ tetrahedra. The cases $i=4$ and $i=5$ require just two and three tetrahedra, respectively. For each $r=1, \ldots, 6$ and $d=r+1, \ldots, 4 r+1$, we computed the change in dimension due to the last step. In all cases it turns out that $\ell_{i}=\delta_{i}$. As a check on our upper bounds, we also directly counted the number of unconstrained points.

Our next example shows that in general the bounds in Theorem 3.1 depend on the order in which the tetrahedral partition is assembled.

Example 8.2. Let $\triangle$ be the partition described in Tab. 3.

Discussion: This partition is obtained by splitting a given tetrahedron into 15 subtetrahedra. It is the trivariate analog of the well-known Morgan-Scott split of a triangle, see [5]. The table lists the coordinates $\left(x_{i}, y_{i}, z_{i}\right)$ of each of the 8 vertices of $\triangle$, and the vertex numbers $\left(v_{0}^{i}, v_{1}^{i}, v_{2}^{i}, v_{3}^{i}\right)$ of each of the 15 tetrahedra in $\triangle$. Tab. 4 shows the results obtained for selected values of $r$ and $d$. The column labeled symdim gives the exact dimension of the splines spaces for the specific choice of vertices given in the table, which corresponds to a high level of symmetry. The column labeled gendim gives the corresponding results for a generic partition obtained by perturbing each of the vertices slightly. We give two sets of upper and lower bounds. The columns labeled $L_{1}(r, d)$ and $U_{1}(r, d)$ correspond to an assembly scheme for which $\tau_{0}=\tau_{5}=\tau_{7}=\tau_{10}=\tau_{26}=1, \tau_{24}=6$, and $\tau_{25}=4$.

The columns labeled $L_{2}(r, d)$ and $U_{2}(r, d)$ correspond to an assembly scheme for which $\tau_{0}=1, \tau_{23}=4, \tau_{24}=6$, and $\tau_{25}=4$. The table shows that the bounds 


\begin{tabular}{|r||r|r|r|r||r|r|r|}
\hline \multicolumn{1}{|c||}{$i$} & $v_{0}^{i}$ & $v_{1}^{i}$ & $v_{2}^{i}$ & $v_{3}^{i}$ & $x_{i}$ & $y_{i}$ & $z_{i}$ \\
\hline 0 & 4 & 5 & 6 & 7 & 14 & 42 & 42 \\
1 & 3 & 4 & 5 & 6 & 14 & 0 & 42 \\
2 & 2 & 4 & 5 & 7 & -14 & 21 & 21 \\
3 & 1 & 4 & 6 & 7 & 0 & 21 & 0 \\
4 & 0 & 5 & 6 & 7 & 2 & 18 & 24 \\
5 & 2 & 3 & 4 & 5 & 2 & 24 & 24 \\
6 & 1 & 3 & 4 & 6 & 6 & 21 & 27 \\
7 & 1 & 2 & 4 & 7 & 4 & 21 & 30 \\
8 & 0 & 3 & 5 & 6 & & & \\
9 & 0 & 2 & 5 & 7 & & & \\
10 & 0 & 1 & 6 & 7 & & & \\
11 & 0 & 1 & 2 & 7 & & & \\
12 & 0 & 1 & 3 & 6 & & & \\
13 & 0 & 2 & 3 & 5 & & & \\
14 & 1 & 2 & 3 & 4 & & & \\
\hline
\end{tabular}

Tab. 3. The tetrahedral partition for Example 8.2.

depend on the assembly order. For this example, the second set of bounds are better.

Our next example gives results for a partition with a hole.

Example 8.3. Let $\triangle$ be the tetrahedral partition described in Tab. 5 .

Discussion: $\triangle$ is a partition of a square-shaped torus into 24 tetrahedra. In Tab. 6 we give results for selected values of $r$ and $d$, both for the symmetric case corresponding to the listed vertices, and for the generic case corresponding to slightly perturbed vertices. In both cases we give bounds corresponding to assembly parameters $\tau_{0}=1, \tau_{19}=4, \tau_{23}=12, \tau_{24}=\tau_{25}=3$, and $\tau_{27}=1$

Our final example involves a partition with a cavity.

Example 8.4. Let $\triangle$ be the partition obtained by removing the tetrahedron numbered 0 in Tab. 3 from the partition described there.

Discussion: Removing the tetrahedron creates a cavity. This partition can be built using the assembly parameters $\tau_{0}=\tau_{5}=\tau_{7}=\tau_{10}=1, \tau_{24}=6$, and $\tau_{25}=3$. In Tab. 7 we give numerical results for selected values of $r$ and $d$, where as before we consider both the generic and symmetric case. For $r=1$ we note that the lower bounds give the correct dimensions for all $d \geq 4$. 


\begin{tabular}{|c|c|c|c|c|c|c|c|}
\hline$r$ & $d$ & $L_{1}(r, d)$ & $L_{2}(r, d)$ & gendim & symdim & $U_{2}(r, d)$ & $U_{1}(r, d)$ \\
\hline 1 & 2 & 10 & 10 & 10 & 11 & 14 & 15 \\
\hline 1 & 3 & 20 & 20 & 20 & 25 & 36 & 39 \\
\hline 1 & 4 & 43 & 43 & 43 & 54 & 81 & 88 \\
\hline 1 & 5 & 104 & 104 & 104 & 113 & 160 & 172 \\
\hline 1 & 6 & 214 & 214 & 214 & 222 & 288 & 306 \\
\hline 1 & 7 & 388 & 388 & 388 & 396 & 480 & 504 \\
\hline 1 & 8 & 641 & 641 & 641 & 649 & 751 & 781 \\
\hline 1 & 9 & 988 & 988 & 988 & 996 & 1116 & 1152 \\
\hline 2 & 3 & 20 & 20 & 20 & 20 & 24 & 26 \\
\hline 2 & 4 & 35 & 35 & 35 & 35 & 51 & 57 \\
\hline 2 & 5 & 56 & 56 & 56 & 56 & 96 & 109 \\
\hline 2 & 6 & 84 & 84 & 84 & 87 & 170 & 193 \\
\hline 2 & 7 & 120 & 120 & 132 & 144 & 284 & 320 \\
\hline 2 & 8 & 203 & 207 & 243 & 252 & 449 & 500 \\
\hline 2 & 9 & 380 & 384 & 420 & 428 & 680 & 748 \\
\hline 3 & 4 & 35 & 35 & 35 & 35 & 39 & 42 \\
\hline 3 & 5 & 56 & 56 & 56 & 56 & 72 & 81 \\
\hline 3 & 6 & 84 & 84 & 84 & 84 & 124 & 143 \\
\hline 3 & 7 & 120 & 120 & 120 & 120 & 200 & 233 \\
\hline 3 & 8 & 165 & 165 & 165 & 167 & 311 & 363 \\
\hline 3 & 9 & 220 & 220 & 220 & 234 & 468 & 543 \\
\hline 3 & 10 & 286 & 286 & 332 & 348 & 682 & 784 \\
\hline 3 & 11 & 364 & 364 & 524 & 534 & 964 & 1096 \\
\hline 3 & 12 & 581 & 593 & 799 & 807 & 1329 & 1494 \\
\hline 3 & 13 & 936 & 948 & 1172 & 1180 & 1792 & 1992 \\
\hline 4 & 5 & 56 & 56 & 56 & 56 & 60 & 64 \\
\hline 4 & 6 & 84 & 84 & 84 & 84 & 100 & 112 \\
\hline 4 & 7 & 120 & 120 & 120 & 120 & 160 & 185 \\
\hline 4 & 8 & 165 & 165 & 165 & 165 & 245 & 288 \\
\hline 4 & 9 & 220 & 220 & 220 & 220 & 360 & 427 \\
\hline 4 & 10 & 286 & 286 & 286 & 287 & 516 & 613 \\
\hline 4 & 11 & 364 & 364 & 364 & 371 & 724 & 857 \\
\hline 4 & 12 & 455 & 455 & 455 & 486 & 995 & 1169 \\
\hline 4 & 13 & 560 & 560 & 640 & 667 & 1340 & 1560 \\
\hline 4 & 14 & 680 & 680 & 926 & 944 & 1770 & 2040 \\
\hline
\end{tabular}

Tab. 4. Results for Example 8.2.

\section{$\S$ 9. Remarks}

Remark 1. Given a tetrahedral partition $\triangle$, let $\mathcal{D}_{d, \triangle}$ be the set of all domain points associated with tetrahedra of $\triangle$, where common domain points are included just once. Then the dimension of the trivariate spline space $\mathcal{S}_{d}^{0}(\triangle)$ is equal to the 


\begin{tabular}{|r||r|r|r|r||r|r|r|}
\hline \multicolumn{1}{|c||}{$i$} & $v_{0}^{i}$ & $v_{1}^{i}$ & $v_{2}^{i}$ & $v_{3}^{i}$ & $x_{i}$ & $y_{i}$ & $z_{i}$ \\
\hline 0 & 0 & 3 & 7 & 15 & -1 & 1 & 0 \\
1 & 0 & 4 & 7 & 15 & -1 & -1 & 0 \\
2 & 0 & 4 & 12 & 15 & 1 & -1 & 0 \\
3 & 0 & 8 & 12 & 15 & 1 & 1 & 0 \\
4 & 0 & 8 & 11 & 15 & -2 & 2 & 0 \\
5 & 0 & 3 & 11 & 15 & -2 & -2 & 0 \\
6 & 0 & 1 & 4 & 12 & 2 & -2 & 0 \\
7 & 1 & 4 & 5 & 12 & 2 & 2 & 0 \\
8 & 1 & 5 & 12 & 13 & -1 & 1 & 1 \\
9 & 1 & 9 & 12 & 13 & -1 & -1 & 1 \\
10 & 1 & 8 & 9 & 12 & 1 & -1 & 1 \\
11 & 0 & 1 & 8 & 12 & 1 & 1 & 1 \\
12 & 1 & 2 & 5 & 13 & -2 & 2 & 1 \\
13 & 2 & 5 & 6 & 13 & -2 & -2 & 1 \\
14 & 2 & 6 & 13 & 14 & 2 & -2 & 1 \\
15 & 2 & 10 & 13 & 14 & 2 & 2 & 1 \\
16 & 2 & 9 & 10 & 13 & & & \\
17 & 1 & 2 & 9 & 13 & & & \\
18 & 2 & 3 & 6 & 14 & & & \\
19 & 3 & 6 & 7 & 14 & & & \\
20 & 3 & 7 & 14 & 15 & & & \\
21 & 3 & 11 & 14 & 15 & & & \\
22 & 3 & 10 & 11 & 14 & & & \\
23 & 2 & 3 & 10 & 14 & & & \\
\hline
\end{tabular}

Tab. 5. The tetrahedral partition for Example 8.3.

the number of points in the set $\mathcal{D}_{d, \triangle}$. This is $n_{V}+(d-1) n_{E}+\left(\begin{array}{c}d-1 \\ 2\end{array}\right) n_{F}+\left(\begin{array}{c}d-1 \\ 3\end{array}\right) n_{T}$, where $n_{V}, n_{E}, n_{F}$, and $n_{T}$ are the numbers of vertices, edges, faces, and tetrahedra in $\triangle$. At the other extreme, the dimension of $\mathcal{S}_{d}^{d}(\triangle)$ is equal to $m\left(\begin{array}{c}d+3 \\ 3\end{array}\right)$, where $m$ is the number of connected components of $\triangle$.

Remark 2. By definition, any shellable tetrahedral partition is homeomorphic to a ball in $\mathbb{R}^{3}$. The simplest example of a nonshellable partition is the partition consisting of two tetrahedra touching at one vertex. The fact that a tetrahedral partition is homeomorphic to a ball does not imply that it is shellable. For an example consisting of 41 tetrahedra and 14 vertices, see Rudin [6].

Remark 3. For the special case of shellable tetrahedral decompositions, the upper bounds given here agree with those given in [1]. Our lower bounds agree for $r=1$, and provide a significant improvement when $r>1$. That paper contains two typographical errors: the summation indices in (10) and (15) there should be $\kappa$ instead of $i$, and the upper limits of the summation should be $k+1$ and $k$ instead of $\kappa+1$ and $\kappa$, respectively. 


\begin{tabular}{|c|c|c|c|c|c|}
\hline$r$ & $d$ & $L(r, d)$ & gendim & symdim & $U(r, d)$ \\
\hline 1 & 2 & 10 & 10 & 12 & 22 \\
1 & 3 & 48 & 48 & 48 & 68 \\
1 & 4 & 144 & 144 & 144 & 176 \\
1 & 5 & 320 & 320 & 320 & 364 \\
1 & 6 & 600 & 600 & 600 & 656 \\
1 & 7 & 1008 & 1008 & 1008 & 1076 \\
1 & 8 & 1568 & 1568 & 1568 & 1648 \\
1 & 9 & 2304 & 2304 & 2304 & 2396 \\
\hline 2 & 3 & 20 & 20 & 22 & 32 \\
2 & 4 & 35 & 35 & 46 & 83 \\
2 & 5 & 93 & 96 & 108 & 176 \\
2 & 6 & 237 & 240 & 256 & 353 \\
2 & 7 & 477 & 480 & 500 & 629 \\
2 & 8 & 837 & 840 & 864 & 1025 \\
2 & 9 & 1341 & 1344 & 1372 & 1565 \\
\hline 3 & 4 & 35 & 35 & 37 & 47 \\
3 & 5 & 56 & 56 & 64 & 104 \\
3 & 6 & 84 & 84 & 112 & 204 \\
3 & 7 & 151 & 176 & 208 & 360 \\
3 & 8 & 351 & 380 & 420 & 623 \\
3 & 9 & 663 & 696 & 744 & 1004 \\
3 & 10 & 1111 & 1148 & 1204 & 1524 \\
3 & 11 & 1719 & 1760 & 1824 & 2204 \\
3 & 12 & 2511 & 2556 & 2628 & 3068 \\
3 & 13 & 3511 & 3560 & 3640 & 4140 \\
\hline 4 & 5 & 56 & 56 & 58 & 68 \\
4 & 6 & 84 & 84 & 92 & 132 \\
4 & 7 & 120 & 120 & 140 & 240 \\
4 & 8 & 165 & 165 & 220 & 405 \\
4 & 9 & 220 & 288 & 356 & 640 \\
4 & 10 & 483 & 560 & 644 & 1006 \\
4 & 11 & 875 & 960 & 1060 & 1509 \\
4 & 12 & 1419 & 1512 & 1628 & 2170 \\
4 & 13 & 2139 & 2240 & 2372 & 3010 \\
4 & 14 & 3059 & 3168 & 3316 & 4050 \\
\hline
\end{tabular}

Tab. 6. Results for Example 8.3.

Remark 4. A shellable partition can be built using only assembly steps of types $0,23,24$, and 25. However, a tetrahedral partition of a set $\Omega$ with holes and/or cavities cannot be shellable. In [3], a tetrahedral partition $\triangle$ is said to have a regular cavity provided that it can be obtained from a larger tetrahedral partition by removing a shellable subpartition $\mathcal{T}$, all of whose vertices are interior vertices 


\begin{tabular}{|c|c|c|c|c|c|}
\hline$r$ & $d$ & $L(r, d)$ & gendim & symdim & $U(r, d)$ \\
\hline 1 & 2 & 10 & 10 & 11 & 15 \\
1 & 3 & 20 & 20 & 25 & 39 \\
1 & 4 & 46 & 46 & 57 & 88 \\
1 & 5 & 112 & 112 & 121 & 172 \\
1 & 6 & 228 & 228 & 236 & 306 \\
1 & 7 & 408 & 408 & 416 & 504 \\
1 & 8 & 666 & 666 & 674 & 780 \\
1 & 9 & 1016 & 1016 & 1024 & 1148 \\
\hline 2 & 3 & 20 & 20 & 20 & 26 \\
2 & 4 & 35 & 35 & 35 & 57 \\
2 & 5 & 56 & 56 & 57 & 109 \\
2 & 6 & 84 & 84 & 91 & 193 \\
2 & 7 & 120 & 144 & 157 & 320 \\
2 & 8 & 242 & 270 & 279 & 500 \\
2 & 9 & 436 & 464 & 472 & 748 \\
\hline 3 & 4 & 35 & 35 & 35 & 42 \\
3 & 5 & 56 & 56 & 56 & 81 \\
3 & 6 & 84 & 84 & 84 & 143 \\
3 & 7 & 120 & 120 & 120 & 233 \\
3 & 8 & 165 & 165 & 168 & 363 \\
3 & 9 & 220 & 224 & 245 & 543 \\
3 & 10 & 286 & 368 & 381 & 784 \\
3 & 11 & 428 & 584 & 593 & 1096 \\
3 & 12 & 718 & 886 & 894 & 1494 \\
3 & 13 & 1108 & 1288 & 1296 & 1992 \\
\hline 4 & 5 & 56 & 56 & 56 & 64 \\
4 & 6 & 84 & 84 & 84 & 112 \\
4 & 7 & 120 & 120 & 120 & 185 \\
4 & 8 & 165 & 165 & 165 & 288 \\
4 & 9 & 220 & 220 & 220 & 427 \\
4 & 10 & 286 & 286 & 289 & 613 \\
4 & 11 & 364 & 364 & 380 & 857 \\
4 & 12 & 455 & 478 & 518 & 1169 \\
4 & 13 & 560 & 712 & 736 & 1560 \\
4 & 14 & 680 & 1036 & 1053 & 2040 \\
\hline
\end{tabular}

Tab. 7. Results for Example 8.4.

of $\triangle$. A tetrahedral partition $\triangle$ of a set $\Omega$ is said to have a regular hole provided that it can be obtained from a larger tetrahedral partition by removing a shellable subpartition $\mathcal{T}$ with the property that if $F$ is a triangular face of a tetrahedron in $\mathcal{T}$ and $F$ lies on the boundary of $\Omega$, then $F \in t_{1} \cup t_{2}$, where 
1) $t_{1}$ and $t_{2}$ are shellable triangulations,

2) the triangles of $t_{1}$ do not touch those in $t_{2}$.

In [3], a tetrahedral partition $\triangle$ is said to be regular provided that one of the following holds:

1) $\triangle$ is shellable, or

2) $\triangle$ can be obtained from a regular tetrahedral partition by creating a regular hole or a regular cavity.

This definition is general enough to include all of the tetrahedral partitions typically used in practice. We conjecture that regular partitions can be built using only assembly steps of types $0,23,24,25$, and 27 .

Remark 5. It was shown in [1] that there exists a tetrahedral decomposition such an arbitrarily small change in the location of the vertices changes the dimension of the corresponding spline space $\mathcal{S}_{d}^{r}(\triangle)$ by a term which is linear in $d$. It follows that any upper and lower bounds on the dimension of $\mathcal{S}_{d}^{r}(\triangle)$ that work for arbitrary partitions and do not take account of the precise locations of the vertices must differ by a term that is at least $\mathcal{O}(d)$. We claim that for $d>2 r$, the upper and lower bounds of Theorem 3.1 satisfy $U(r, d)-L(r, d)=\mathcal{O}(d)$, and thus can be considered to be asymptotically optimal. To see this, we note that the upper bound is $U(r, d)=n_{d}-n_{\text {tips }}$, where $n_{\text {tips }}$ is the number of points that are tips of smoothness conditions. By the discussion in Sect. $6, L(r, d) \geq n_{d}-n_{\text {cond }}$, where $n_{\text {cond }}$ is the number of conditions imposed by smoothness. Thus $U(r, d)-L(r, d) \geq n_{\text {cond }}-n_{\text {tips }}$. When $d>2 r$, a point can be the tip of more than one smoothness condition only if it lies in a tube $t_{r}(e)$ around an edge of $\triangle$, and it follows that $n_{\text {cond }}-n_{\text {tips }}=\mathcal{O}(d)$. This proves the claim.

Remark 6. The java software that we used for testing our bounds is available at http://www.math.utah.edu/ $\mathrm{pa} / 3 \mathrm{DMDS} /$. It can be used as an applet directly from the web page, or can be downloaded and used offline.

Remark 7. Explicit formulae for the dimension of $\mathcal{S}_{d}^{1}(\triangle)$ for $d \geq 8$ were established in [2] for generic shellable tetrahedral partitions. Comparing with Theorem 7.1, it is easy to see that for such partitions the dimension of $\mathcal{S}_{d}^{1}(\triangle)$ is equal to the lower bound in (7.1).

Remark 8. Recently Lau [4] gave a lower bound for the dimension of $\mathcal{S}_{d}^{r}(\triangle)$ for tetrahedral partitions of simply connected domains. His lower bound differs from our lower bound in Theorem 7.1 in two ways. First, it is missing the term involving $V_{I}$, and thus is generally much too low. For example, for the partition of Example 8.2 and for the range of $r$ and $d$ shown in Tab. 4, it gives negative numbers for almost all cases. On the other hand, his bound contains an additional nonnegative term which takes account of the geometry of faces surrounding interior edges. We have not attempted to take account of geometry here. 
Acknowledgement: The first author has benefited from useful conversations with Stew Ethier about some of the combinatorial aspects of the formulae in Sect. 5.

\section{References}

1. Alfeld, P., Upper and lower bounds on the dimension of multivariate spline spaces, SIAM J. Numer. Anal. 33 (1996), 571-588.

2. Alfeld, P., L.L. Schumaker, and W. Whiteley, The generic dimension of the space of $C^{1}$ splines of degree $d \geq 8$ on tetrahedral decompositions, SIAM J. Numer. Anal. 30(3) (1993), 889-920.

3. Lai, M.-J. and L. L. Schumaker, Spline Functions on Triangulations, Cambridge University Press, Cambridge, 2007.

4. Lau, W. W., A lower bound for the dimension of trivariate spline spaces, Constr. Approx. 23 (2006), 23-31.

5. Morgan, John and Ridgway Scott, A nodal basis for $C^{1}$ piecewise polynomials of degree $n \geq 5$, Math. Comp. 29 (1975), 736-740.

6. Rudin, M. E., A unshellable triangulation of a tetrahedron, Bull. Amer. Math. Soc., 64, 1958, 90-91. 